\title{
Balkanologie
}

Balkanologie Revue d'études pluridisciplinaires

Vol. I, n 2 | 1997

Volume I Numéro 2

\section{Prédilections universitaires pour les questions balkaniques}

\section{Thanos Veremis}

Traducteur : Patrick Michels

\section{(Q) OpenEdition \\ Journals}

Édition électronique

URL : http://journals.openedition.org/balkanologie/224

DOI : 10.4000/balkanologie.224

ISSN : 1965-0582

Éditeur

Association française d'études sur les Balkans (Afebalk)

Édition imprimée

Date de publication : 1 décembre 1997

ISSN : 1279-7952

\section{Référence électronique}

Thanos Veremis, «Prédilections universitaires pour les questions balkaniques », Balkanologie [En ligne], Vol. I, n 2 | 1997, mis en ligne le 02 juin 2008, consulté le 17 décembre 2020. URL : http:// journals.openedition.org/balkanologie/224; DOI : https://doi.org/10.4000/balkanologie.224

Ce document a été généré automatiquement le 17 décembre 2020.

(c) Tous droits réservés 


\title{
Prédilections universitaires pour les questions balkaniques
}

\author{
Thanos Veremis \\ Traduction : Patrick Michels
}

\section{RÉFÉRENCE}

Banac (Ivo), The National Question in Yugoslavia: Origins, History, Politics, Ihaca / London : Cornell Universtity Press, 1993 (3rd printing, copyright 1984), 452 p.

Brown (J. F.), Nationalism, Democracy and Security in the Balkans, Aldershot : Dartmouth Publishing, 1992, x-205 p.

Dragnich (Alex N.), Serbs and Croats. The Struggle in Yugoslavia, New York / London :

Harcourt Brace Jovanovich, 1992, xxi-202 p.

Glenny (Misha), The Fall of Yugoslavia. The Third Balkan War, London : Penguin, 1993 (3rd

ed.), xiv-258 p.

Smith (Anthony D.), National Identity, London : Penguin Books, 1991, 227 p.

\section{NOTE DE L'ÉDITEUR}

Ces notes de lectures ont paru dans European History Quarlerly, 24 (4), October 1994. Elles ont été traduites de l'anglais avec l'aimable autorisation de l'auteur par Patrick

Michels.

1 À l'exception de National Identity d'Anthony Smith, ce compte-rendu concerne des travaux qui, à des degrés divers, dépendent des affiliations nationales ou des convictions personnelles de leurs auteurs. Ainsi, Alex Dragnich ne cache pas sa loyauté envers la Serbie, Ivo Banac n'aborde sa genèse de la Yougoslavie qu'avec un penchant croate, Jim Brown trahit sa préférence pour des prédictions avidement consommées par les décideurs politiques américains, et le témoignage de Misha Glenny est teinté de son humanisme libéral. Aussi compréhensibles, ou même recommandables, que 
puissent être leurs prédispositions, il serait juste de supposer que l'objectivité scientifique dans ces exemples n'a fait aucun progrès en comparaison du prototype fourni par Thucydide. Ceci dit, ils sont bien évidemment différents dans tous les autres aspects, dont leur valeur et leur qualité scientifiques. Ainsi, Banac a réalisé un travail de référence, dont l'artisanat diligent ne peut être ignoré ni par l'ami ni par l'adversaire, et la descente de Glenny dans l'enfer yougoslave actuel est une chronique que les historiens futurs devront consulter.

2 Banac et Dragnich constituent une véritable étude de cas: ils montrent que deux travaux traitant de sujets connexes peuvent différer dans presque toutes leurs conclusions lorsque leurs prémisses sont influencées par des préjugés nationaux. Les deux auteurs sont professeurs dans des universités américaines, ils sont néanmoins indubitablement croate et serbe dans leurs loyautés.

Dans l'ensemble, Banac est un artisan supérieur. Son hypothèse, que la Yougoslavie était, dès le début de sa fondation, une création superficielle destinée à s'effondrer, a été élaborée dans un livre ayant suscité un travail monumental qui traite de deux années et demi (1918-1921) de l'histoire du Royaume des Serbes, Croates et Slovènes, constituant, selon l'auteur, «le prélude qui contient toutes les graines des désordres à venir». Bien qu'anti-marxiste notoire, Banac estime que toute l'entreprise d'unification des Slaves du sud était fondée sur la conscience erronée d'idéologies élaborées par des intellectuels ne tenant aucun compte des idéologies nationales, mutuellement exclusives, contraires à l'unification.

4 Les délibérations des Slaves du sud qui ont abouti à leur unification à la fin de la Première Guerre mondiale est au cœur du livre de Banac. Trois groupes ont pris part à ce processus : le gouvernement serbe en exil, l'organisation des Slaves du sud émigrés dans les pays de l'Entente, et les dirigeants politiques des Slaves du sud qui demeuraient en Autriche. Le premier ministre de Serbie, Nikola Pašić, était le moteur des négociations. Il devait satisfaire les dirigeants croates qui souhaitaient l'unification sud-slave (et non pas l'indépendance croate), mais sur la base d'une égalité nationale avec la Serbie. Une des pressions exercée sur les Croates pour conclure l'unification était le Traité secret de Londres entre l'Entente et l'Italie (26 avril 1915) qui donnait à celle-ci les territoires côtiers de la Croatie et de la Slovénie, ainsi que les îles. L'accord de Corfou du 20 juillet 1917 fut conclu entre le gouvernement serbe et le "Comité yougoslave" formé d'hommes politiques anti-habsbourgeois et slovènes en exil. Ce document proclamait la détermination des Serbes, des Croates et des Slovènes de former un État uni qui serait une monarchie constitutionnelle, démocratique et parlementaire, dirigée par la dynastie serbe Karađorđević. En octobre 1918 les délégués des partis croates, serbes et slovènes en faveur d'un État sud-slave indépendant formèrent leur corps représentatif, le "Conseil national des Slovènes, Croates et Serbes". En l'absence d'une armée et, plus encore, d'un large soutien populaire, le "Conseil national" était harcelé par les opérations navales italiennes et l'agitation sociale interne. «Isolé, ignoré par les Alliés, ses hommes sur la côte réprimés par les Italiens, le conseil national était de plus en plus forcé de rechercher l'intervention serbe » (Banac, 131).

5 Lors de la réunion de Genève du 6 novembre 1918 entre le gouvernement serbe, son opposition, le "Conseil national" et le "Comité yougoslave", Pašić consentit à reconnaître le "Conseil national" de Zagreb comme le gouvernement légitime de tous les Slaves du sud (incluant les Serbes de Croatie, de Slovénie et de Bosnie-Herzégovine) 
vivant dans le territoire de la monarchie austro-hongroise. Le 9 novembre, les conditions devant mener à un État unifié des Serbes, Croates et Slovènes furent acceptées.

6 Stjepan Radić et ses adeptes paysans devinrent les plus fervents adversaires de l'unification. Initialement, Radić proposa une confédération dirigée par trois régents (le roi serbe, le parlement croate et le président du conseil national slovène). Des 28 membres de la délégation du "Conseil national", il fut le seul à voter contre la proposition d'unification. Ce fut le prélude à ses activités politiques antiserbes qui aboutirent à son assassinat en 1928.

7 À la différence de Banac, qui cherche systématiquement les motifs cachés des Serbes dans l'unification, Dragnich se fait le champion de l'innocence de la Serbie. "Les puissances de l'Entente avaient offert de récompenser leur allié serbe en promettant qu'après la guerre la Serbie obtiendrait la Bosnie-Herzégovine et d'autres parties de l'Autriche-Hongrie peuplées par des Serbes, ainsi qu'une grande partie de la Dalmatie » (Dragnich, 21). Selon lui, le gouvernement serbe a refusé les offres de l'Entente d'une plus grande Serbie. Dans sa déclaration de Niš de décembre 1914, Pašić annonça que l'aspiration de la Serbie était de libérer les Slaves du sud. Cependant, en 1917, les députés Slaves du sud de l'Autriche-Hongrie pressèrent les provinces peuplées de Croates, de Slovènes et de Serbes de s'unir sous le sceptre de la dynastie des Habsbourg. Pašić fut ainsi forcé de compter sur les Croates expatriés pour signer la déclaration de Corfou. Se défendant des accusations prétendant que la Serbie se destinait à dominer les Slaves du sud, il affirma que la Croatie et la Slovénie seraient libres de choisir entre l'unification et la formation de petits États comme dans le passé. De plus, il promit de ne pas invoquer la déclaration de Corfou si elle ne correspondait pas à leurs voeux.

8 La déclaration de Genève de 1918 reflétait les desirata du "Conseil national" et vraisemblablement ceux des habitants de Croatie et de Slovénie, mais elle fut rejetée par le cabinet serbe (encore en exil dans l'île de Corfou) et le "Conseil national" de Zagreb.

9 Cependant, l'effondrement de l'empire austro-hongrois amena le chaos dans ses provinces slaves du sud. Tandis que les Italiens réitéraient leurs revendications sur la Croatie et que les autorités de Zagreb n'avaient aucun pouvoir pour imposer l'ordre aux paysans rebelles, les forces serbes avaient libéré Belgrade et avaient fermement installé Pašić aux commandes du pays. Le $1^{\text {er }}$ décembre 1918, le roi Aleksandar acceptait les conditions des représentants du "Conseil national", et, ainsi, le Royaume des Serbes, Croates et Slovènes fut enfin créé.

10 Il semble que le moment critique dans les négociations serbo-croates survint après l'accord de Genève. Banac croit que les politiciens de Zagreb s'étaient montrés conciliants, et que Svetozar Pribićevićpoliticien agissant comme un courtier du pouvoir dans la capitale croate, entretenait des liens secrets avec Belgrade. C'est pourquoi Pašić fut en mesure de se retirer du courant confédéral lors de l'accord de Genève avec un minimum de protestation du côté croate. Dragnich, quant à lui, attribue le changement d'esprit croate (d'un ferme confédéralisme à l'acceptation de l'État unitaire) à des circonstances indépendantes de la volonté des autorités croates: la Croatie était occupée par des forces italiennes supérieures en nombre, tandis que la Voïvodine, le Monténégro et la plus grande partie de la Bosnie-Herzégovine s'étaient prononcés en faveur de l'union avec la Serbie. 
11 La vérification de l'allégation de Banac, que l'échec de l'expérience yougoslave était «structurellement inévitable », nécessiterait l'analyse de toute la période de l'entredeux-guerres, ce qui est au-delà de l'étendue de son livre. Cependant, le portrait qu'il dresse de Radić semble indiquer qu'il considère les petits États indépendants comme une alternative qui aurait été viable, malgré les événements qui s'y opposaient, notamment la volonté expansionniste de l'Italie. L'analyse de Banac des deux redoutables adversaires en compétition pour l'avenir de la Yougoslavie, Pašić et Radićn'est, en aucune façon, impartiale. Radić est présenté avec affection: «romantique, et pourtant pragmatique, Stjepan Radić illuminait les cieux croates comme l'éclair lumineux d'un météore » (Banac, 226) ; alors qu'il était un idéologue, un écrivain ou un orateur passable, voire même un démagogue. Sa grammaire était atroce, et sa connaissance dérisoire de l'histoire et de la littérature était le sujet de nombreuses plaisanteries (Banac, 158). De surcroît, Banac utilise le témoignage d'un universitaire connu pour son attitude négative envers tout ce qui était serbe: "Seton Watson l'abhorrait et avertit qu'après l'unification, la "Barbe" [Pašić] jouerait "simplement à son vieux jeu d'intrigues balkaniques" " (Banac, 159). Comme c'est le cas avec tant d'autres choses, Banac et Dragnich ne sont pas d'accord sur la présence ou l'absence de volonté des communistes envers l'union yougoslave.

12 Si les préjugés nationaux de Banac sont compréhensibles, l'analyse autoritaire de J. F. Brown l'est moins. Son Nationalisme, démocratie et sécurité dans les Balkans est empli de jugements de valeur s'adressant sans aucun doute à un lectorat qui partage avec lui certains principes fondamentaux. Son affirmation qu'» aucun pays de l'Europe de l'est, sauf la Tchécoslovaquie, ne connut de démocratie viable avant la Seconde Guerre mondiale» (p. 111) est une erreur, à moins que des normes fermes de régime démocratique soient posées. Si ces normes devaient en exclure l'Europe de l'est dans sa totalité, elles devraient certainement en exclure également une bonne partie de l'Europe occidentale. La conviction de Dragnich que «dans la décennie précédant la Première Guerre mondiale, la Serbie avait un système politique démocratique qui grosso modo correspondait à celui de la France, des Pays-Bas et du Danemark contemporains " (p.5) est, certes, une exagération, mais elle correspond au fait que les quinze premières années de notre siècle n'étaient pas néfastes aux régimes parlementaires en général.

22 ans auparavant (Bulgaria Under Communist Rule [London, 1970]), Brown se montrait plus disposé à l'égard du régime de Zhivkov qu'il ne le fut après-coup : «les progrès rapides dans la santé publique, l'instruction, l'existence d'une plus grande opportunité - seul un adversaire aveugle du communisme mettrait en doute l'autosatisfaction officielle sur ces éléments» (p. 302); "Zhivkov, un homme modeste et bien intentionné, croit probablement aux sentiments et aux propositions de réforme qu'il a si souvent exprimés (...), les réformes en Bulgarie, au contraire de celles de Hongrie ou plus récemment de la Roumanie de Ceaucescu, ont souvent semblé avoir quelque chose d'accidentel...» (p. 303). On n'attend pas des historiens qu'ils disposent de pouvoirs prophétiques, mais les prédictions et les prescriptions de Brown dans le dernier quart de son livre sont déjà contestables. Les deux vastes "coalitions opposées" de la chrétienté orthodoxe et de l'islam dans les Balkans ne parait pas être fondée. Ni la propagation du conflit yougoslave ou l'éruption de la guerre entre la Serbie et l'Albanie. Dans son analyse systématique de la crise yougoslave, Brown échoue à mettre en évidence un point qui a été expliqué avant que son livre ne soit publié: si la dissolution de la Yougoslavie est survenue parce que les Croates, les Slovènes et les 
Musulmans refusaient de vivre dans un État avec un élément serbe prépondérant, les cinq États résultant ont reproduit le même problème dans leurs propres territoires échangeant une nation dominante contre une autre. Ce que la communauté européenne a réussi, en reconnaissant les nouveaux États sur la base de la prépondérance nationale, est de légitimer la base nationale d'États unitaires.

Misha Glenny n'a pas manqué cette clef vitale à la compréhension de la crise yougoslave. Il critique sévèrement aussi bien les acteurs que ceux qui les légitiment. Son argument final est de force: "l'effondrement yougoslave fournissait à la communauté internationale une occasion de saisir l'essence du problème politique régional : la question des minorités et des États-nations » (p. 241). Cette simple vérité a échappé à l'attention des faiseurs de politique européens et de leur conseillers universitaires. La règle de la majorité dans chaque communauté peut seulement être efficace lorsque le consensus a été fixé sur des principes fondamentaux de coexistence entre des majorités et des minorités. C'était clairement absent dans presque chaque République yougoslave à la veille de la reconnaissance : «la sentence de mort pour la Bosnie-Herzégovine fut signée au milieu de décembre 1991 lorsque l'Allemagne annonça qu'elle reconnaîtrait la Slovénie et la Croatie inconditionnellement le 15 janvier $1992 »$ (p. 163). Au lieu d'essayer au moins de dupliquer la structure fédérale de la Yougoslavie dans leurs propres territoires afin d'apaiser leurs considérables minorités, les dirigeants des nouvelles républiques commettaient la folie d'établir des États unitaires après la reconnaissance.

15 La volonté d'indépendance se propageait comme un incendie de forêt.

Chaque région de Yougoslavie était devenue la proie d'une lèpre politique. Le concept d'État devenait confus et bien que le droit à l'autodétermination était entièrement légitime, les nations de Yougoslavie étaient en danger de s'émietter elles-mêmes à un degré tel que les Balkans en tant qu'espace économique et social trouverait difficilement un mode de fonctionnement (Glenny, 129).

16 Une fois qu'il était devenu clair qu'il s'agissait du chacun pour soi sur les territoires contestés, le carnage devenait inévitable. Naturellement, les plus puissants des bouchers étaient responsables de la plus grande part des massacres.

17 À l'exception de Banac (pp. 307-328), qui connaît bien le sujet, les autres auteurs commentés ici ont commis une variété d'erreurs factuelles concernant l'ancienne République yougoslave de Macédoine. Dragnich pense faussement que la Serbie obtint la plus grande partie de la Macédoine ottomane (Dragnich, 192), et Glenny croit que la constitution de cet État prévient toute revendication territoriale sur ses voisins (Glenny, 72). Le préambule de la constitution actuelle, cependant, fait clairement référence au document de l'ASNOM, fondateur de la République socialiste en 1944, qui édicte : "Macédoniens en Bulgarie et en Grèce... L'unification du peuple macédonien dépend de votre participation ... $»^{1}$.

Il est très difficile de comprendre la réapparition des identités nationales qui empoisonnent la Yougoslavie sans placer le phénomène dans une perspective théorique. Le livre d'Anthony Smith, National Identity, utilise les outils de la sociologie historique pour tracer les origines et étudier le mécanisme de l'identité nationale. Son livre contient une analyse très perspicace des antécédents pré-modernes des nations modernes et présente les composants culturels importants de l'identité ethnique. Une des questions que pose le livre est : « que-ce qui rend les multiples identités collectives et loyautés compatibles ou conflictuelles?». Autrement dit, qu'est-ce qui fait que les 
loyautés concentriques opèrent en Europe occidentale et aux États-Unis et échouent en Europe de l'est? Dans son analyse de l'expérience supra-nationale, Smith fait un excellent usage du paradigme yougoslave. Selon l'auteur, la Yougoslavie était bâtie autour deux concepts: une fédération de nations et une expérience culturelle et historique commune ; «mais, ainsi que ses idéologues eux-mêmes l'ont concédé, c'était moins l'histoire politique qui tenait les Slaves du sud ensemble que des langues analogues et la proximité géographique, ainsi que, peut-être, l'occupation étrangère " (Smith, 147).

19 En fait, les histoires séparées des Slovènes, des Croates et des Serbes avaient suggéré l'espoir que l'expérience yougoslave devienne un modèle de la "nation transcendée" sous la forme d'une fédération de nations. Cela malheureusement ne devait pas être. Ainsi que Banac et Dragnich le suggèrent, une fois les occupations étrangères révolues, les particularismes nationaux ont fait surface, faisant appel à la vengeance.

\section{NOTES}

1. Le problème "macédonien" a confondu beaucoup d'écrivains dans le passé. Le journaliste Robert Kaplan dans ses Balkan Ghosts, A Journey Through History (New York, St. Martin's Press 1993) cite John Reed (The War in Eastern Europe, New York, Charles Scribner's Sons, 1916) en soulignant son accord avec ses propos :

«La question macédonienne a été la cause de chaque grande guerre européenne dans les 50 dernières années, et jusqu'à ce qu'elle ait été réglée, il ne pourra y avoir de paix, que ce soit dans ou en dehors des Balkans. La Macédoine est le plus effroyable mélange de races jamais imaginé. Turcs, Albanais, Serbes, Roumains, Grecs et Bulgares vivaient côte à côte sans se mêler - et vivent ainsi depuis les jours de St. Paul (sic)», p. 52. 